\title{
Bevacizumab in Wet AMD treatment: A tribute to the thirteen years of experience from the beginning of the anti-VEGF era in Romania
}

\author{
HORIA T. STANCA ${ }^{1}$, SIMONA STANCA ${ }^{2}$, BOGDANA TABACARU ${ }^{1}$, \\ MADALINA BORUGA ${ }^{3}$ and FLORIAN BALTA ${ }^{1}$ \\ Departments of ${ }^{1}$ Ophthalmology and ${ }^{2}$ Pediatrics, 'Carol Davila' University of Medicine and Pharmacy, 050474 Bucharest; \\ ${ }^{3}$ Department of Pharmacy, 'Victor Babes' University of Medicine and Pharmacy, 300041 Timisoara, Romania
}

Received April 22, 2019; Accepted July 26, 2019

DOI: $10.3892 /$ etm.2019.7858

\begin{abstract}
This study aimed to identify and describe anatomical and functional changes on short (1-3 months) and medium (6-12 months) term after intravitreal injections of bevacizumab (Avastin, Genentech) in patients with choroidal neovascularization $(\mathrm{CNV})$ in the context of exudative form of age-related macular degeneration (AMD). We performed a retrospective, analytical, interventional study, based on a series of cases with exudative form of AMD, which also comprised a prospective component related to the inclusion and treatment of the patients with a very new interventional method for that time (2006) and the follow-up of the effects of intravitreal injection of bevacizumab $(1.25 \mathrm{mg})$ therapy in three monthly doses for short (1-3 months) and medium (6-18 months) periods of time. The follow-up of these patients was made by determining visual acuity (VA) as best corrected visual acuity (BCVA) at baseline and at every visit, slit lamp examination with contact or noncontact lenses each time, and optical coherence tomography and/or angiofluorography, applied only for certain patients, at various times of the study. In total, 376 intravitreal injections were administered to 117 eyes of 96 patients. The VA improved in the assessment of 3 months in 77 eyes (66\%), either subjective (by the patient) or objectively quantified (by the physician). In 40 eyes (34\%), there was no change in VA. In patients for whom optical coherence tomography could be performed, a significant reduction of the macula's thickness was found. The use of bevacizumab in subretinal neovascular membrane treatment is effective and safe on short and medium term, with the improvement of BCVA and reduction of macular edema in a significant number of cases.
\end{abstract}

Correspondence to: Dr Simona Stanca, Department of Pediatrics, 'Carol Davila' University of Medicine and Pharmacy, Str. Eroilor Sanitari 8, Sector 5, 050474 Bucharest, Romania

E-mail: simonastanca@yahoo.com

Key words: bevacizumab, central foveal thickness, visual acuity, wet AMD, choroidal neovascularization

\section{Introduction}

Macular degeneration represents the main cause of severe visual impairment in patients over 60 years of age in industrialized countries (1). The prevalence of macular degeneration in people of $40-49$ years is $2.1 \%$, rising dramatically to $35 \%$ in the population aged over 80 years (2).

The severe deterioration of central vision occurs mainly in the exudative form of age-related macular degeneration (AMD) (3), as a result of the formation of pathological choroidal neovessels - choroidal neovascular membranes (CNVM) that induce structural and functional changes in the macular region (4).

Exudative AMD dominates the etiologic spectrum of conditions involving CNVM; therefore, our study focused on the current therapeutic options for the choroidal neovascular membranes in the wet form of AMD.

In advanced stages of AMD, CNVM can cause irreversible loss of central vision to the level of 'legal blindness'. This 'blindness', though not complete (there is a remaining peripheral visual field), reduces the individual's adaptation to the environment, making it impossible to read, drive, recognize facial features of people around them, thus altering the quality of life and autonomy of those affected (4).

Until 2006, the methods of treatment for neovascular choroidal pathology differed from the prescription of prophylactically antioxidant medication, with no effect on the existing membranes, to the 'heroic' surgery that tried to extract the membranes under the retina and possibly 'relocate' the retina, by turning it on healthy pigment epithelium areas, with sometimes ambiguous effects. At this point, because of a new class of drugs, namely vascular endothelial growth factor blockers (anti-VEGF), we have entered a new therapeutic era in the management of these diseases. Thirteen years after having started the intravitreal anti-VEGF treatment in Romania, we are reviewing the steps, the results and the changes in our daily practice.

\section{Materials and methods}

The study was performed based on a number of cases diagnosed with exudative AMD, the inclusion criteria being the 
presence of choroidal neovascularization in the context of AMD, evidenced clinically and on OCT images. Exclusion criteria consisted in the absence of clinical and angiofluorographic exudation features and in the presence of excessive fibrous components. The epidemiological strategy was an analytical, interventional case series without a control group, with a prospective component, which was the introduction of new therapeutic methods (intravitreal injection of bevacizumab) in a pathology (an exudative type of age-related macular degeneration) where treatment solutions were mostly ineffective. The functional and anatomical effectiveness of this treatment was followed-up over short (1-3 months) and medium (6-18 months) periods.

In October 2006, we started to perform the first intravitreal bevacizumab injections. At the beginning of this therapy, its legal aspect was uncertain ('off-label' use of bevacizumab drug - outside the therapeutic indications that were safe) (5). The medium and long-term results were unknown; there was no available data regarding possible systemic side effects of the substance. Regarding the 'off-label' status of this medication, we followed the American model, elaborating a well-informed consent, based on the content used overseas. This study adhered to the tenets of the Declaration of Helsinki and was approved by the Ethics Committee of our hospital, 'Prof. Dr. Agrippa Ionescu' Clinical Emergency Hospital. Written informed consent was obtained from all patients before the examinations and surgeries were performed. As we obtained favorable results in patients with low VA, we began to use intravitreal bevacizumab in patients with the same disease (neovascular AMD), but with better visual acuity (VA) (best VA for initiating treatment was 20/40-0.5). When included in the treatment group, each patient was evaluated for the best corrected visual acuity (BCVA) with Snellen chart and they underwent a complete eye examination, which included measurement of intraocular pressure, refractometry and biomicroscopy of the anterior and posterior pole. Most times, especially at the beginning of the study, the main element that allowed the patient to have access to the treatment algorithm was slit lamp examination with Volk Centralis Direct contact lens. Where clinical macular edema, exudative elements and/or subretinal haemorrhage were found, we used bevacizumab without imaging confirmation. In cases where the fibrovascular component was predominant or the subfoveal changes did not show clinical signs of exudation, we performed FA and/or OCT in order to specify the diagnosis. Central retinal thickness was measured in patients who performed OCT with ' 5 - $\mathrm{mm}$ raster line scans' for patients examined with ZeissStratus model and '6-mm raster line scans' for the investigations with Topcon 3D 1000. Fluoresceine angiographies were performed mainly in patients from the first part of the study group because OCT examination was less accessible at that time. Afterwards, FA was used only as a method to determine the leakage site (extravasation of fluid) at certain period of times after the end of therapy, when there was clinical doubt or VA tended to change.

All patients had previously received antioxidant medication administered orally. The therapeutic protocol consisted of intravitreal administration of bevacizumab $1.25 \mathrm{mg} / 0.05 \mathrm{ml}$ every four weeks in three sessions for each of the eyes of the patients in the study. Each eye was considered a separate entity. Increasing the number of injections (to 4 or 5 injections) over the standard, a protocol was performed to cases which showed clinical and functional improvement after 3 months of treatment. However, relapses followed at intervals varying between at least 3 months and at most 12 months. Patients were clinically reviewed by retinal imagistic after 1 month and 3 months. Some patients continued to be monitored at 6, 12 and 18 months. This was the first study performed in Romania regarding the treatment of wet AMD using bevacizumab. We intend to show our first results from the beginning of the anti-VEGF era to the international ophthalmologic community, in order to demonstrate that our experience, which started in 2006, is consistent with all the data from the literature and our approach, which was not influenced by other authors, can be superimposed on the standard protocols nowadays.

Patients. There were 376 intravitreal injections to 117 eyes [67 left eyes (57.2\%) and 50 right eyes (42.8\%)] from 96 patients [59 males (61.45\%) and 37 females (38.55\%)], starting October 2006. A total of 21 patients had both eyes treated. Patients were classified into three age groups: 55-65 years (average age 62.4 years, 18 patients), 65-75 years (average age 71.7, 37 patients) and over 75 (average age 82.3 years, 41 patients). The average age was 72.1 years.

Treatment protocol. The three 'standard' injections were administered to all patients. Among the 117 treated eyes, 13 received four injections and six eyes received five injections, in addition to standard injections. The criteria by which treatment protocol was supplemented will be discussed further. The structure of the injected study group was as follows: 96 patients: 21 treated in both eyes (OU), 42 eyes, 126 injections; 75 treated in one/single eye (SE), 225 injections. Total number of injections performed, 376 (average number of injections performed/eyes, 3.2).

Follow-up. The patients were functionally monitored, being evaluated with the Snellen chart for central visual acuity (VA). We have opted to express it as decimal VA, because it is simply and easily understood by the patient and it is easier to convert it in the minimum visual resolution angle logarithm (logMAR), a universally accepted mathematical unit in statistical calculations.

The follow-up of retinal structural changes involved performing either optical coherence tomography, which allowed the assessment of macular thickness variations (i.e. the degree of macular edema resolution) or fluorescein angiographies, which verified the degree of activity of choroidal neovascular membranes according to the presence or absence of the extravasation/diffusion fluid (leakage).

Central visual acuity. Central visual acuity was measured at baseline and then after one month, 2 months and 3 months. These data were obtained for all treated eyes (100\% of cases). For some patients, VA follow-up could also be done after 6 months, 12 months and 18 months. Tailure to follow-up the medium controls (6-12/18 months) of patients was determined both by a lower compliance and by old age associated with other systemic diseases, which reduced the addressability to the ophthalmologist. 
Table I. Correlation of the study group patients without change in VA with subjective elements changes.

\begin{tabular}{|c|c|c|c|c|c|}
\hline Group & $\begin{array}{l}\text { Perception } \\
\text { of color }\end{array}$ & $\begin{array}{l}\text { Delimitation of } \\
\text { the contours }\end{array}$ & $\begin{array}{l}\text { The decrease of } \\
\text { central 'darkness' }\end{array}$ & $\begin{array}{l}\text { Reduction of } \\
\text { image distortion }\end{array}$ & $\begin{array}{l}\text { No. of } \\
\text { patients }\end{array}$ \\
\hline $\mathrm{A}(0.01)$ & 2 & 1 & 5 & 2 & 11 \\
\hline B $(0.02)$ & 4 & 3 & 5 & 2 & 16 \\
\hline $\mathrm{C}(0.04)$ & 5 & 3 & 4 & 4 & 8 \\
\hline $\mathrm{D}(0.06)$ & 1 & 0 & 1 & 1 & 2 \\
\hline $\mathrm{E}(0.1)$ & 1 & 1 & 1 & 1 & 2 \\
\hline $\mathrm{F}(0.2)$ & 1 & 0 & 0 & 0 & 1 \\
\hline $\mathrm{G}(0.3)$ & 0 & 0 & 0 & 0 & 0 \\
\hline $\mathrm{H}(0.5)$ & 0 & 0 & 0 & 0 & 0 \\
\hline Total & 14 & 8 & 16 & 10 & 40 \\
\hline
\end{tabular}

We identified eight groups of patients according to the initial value of VA (assessed with the best optical correction): Group A: Initial VA $0.01(0.5 / 50), 26$ eyes; Group B: Initial VA 0.02 (1/50), 43 eyes; Group C: Initial VA 0.04 (2/50), 26 eyes; Group D: Initial VA 0.06 (3/50), 8 eyes; Group E: Initial VA 0.1 (5/50), 7 eyes; Group F: Initial VA 0.2, 4 eyes; Group G: Initial VA 0.3, 1 eye; Group H: Initial VA 0.5, 2 eyes.

The number of patients available for follow-up was: 76 eyes from 63 patients after 6 months, 39 eyes from 28 patients after 9 months, 31 eyes from 21 patients after 12 months and 23 eyes from 19 patients after 18 months.

From the initial study groups, only some patients remained to be followed-up. Thirteen eyes received four injections and six eyes received five injections. Reinjections were performed in cases where a favorable initial response had been found after the first three injections of the therapeutic drug, but followed by a functional relapse (reduction in VA) doubled by exudative phenomena (neovascular membranes' activity marker). Therefore, it was decided to resume therapy in those cases, this time according to the circumstances and adapting, that is injecting bevacizumab not following a scheme, but observing the clinical and paraclinical evolution of the patient - the actual PRN (pro re nata) scheme.

VA measurement involves a subjective component, the patient's response, but in controlled and standardized conditions (Snellen chart, standard lighting conditions). In some patients, we did not detect favorable changes of central visual acuity. However, many patients with unchanged VA reported an improvement in vision. We could not quantify this aspect; therefore, we were unable to integrate it into a statistical analysis. We noted the main favorable elements reported by the patients 3 months after injection (Table I). Among the 117 eyes treated, after 3 months 40 of them (34\%) presented no VA changes.

Central retinal thickness (foveal thickness). Central thickness changes were determined by optical coherence tomography (OCT). Among the 96 patients (117 eyes), 42 patients (43\%) (51 eyes) (43\%) could perform imaging investigation such as angiofluorography and/or OCT. Among these, 51 eyes could be monitored by OCT at baseline and after 3 months from the first injection (Table II).
Table II. Structure of the remaining group of patients that was imagistically followed-up on mid-term.

\begin{tabular}{lcc}
\hline Period & Eyes & OCT performed \\
\hline 6 months & 76 & 9 \\
9 months & 39 & 11 \\
12 months & 29 & 9 \\
18 months & 23 & 3 \\
\hline
\end{tabular}

Among the patients remaining for follow-up after the required period of 3 months, only some of them underwent imaging investigation. Actually, OCT and/or AFG were performed only in patients who had been reinjected, several of whom were monitored by OCT 3 months after reinjection.

Activity signs of choroidal neovascularization. The existence and/or persistence of signs of choroidal neovascular membrane activity, expressed by 'leakage' - extravasation and diffusion of fluid, were followed-up by fluorescein angiography. A minority of patients performed FA - in cases of diagnostic doubt, or those at baseline when the tomographic examination was not available. Numerical data do not provide a coherent statistical overview of the treated group evolution and, therefore, it will not be used.

Statistical analysis. The statistical analyses have been performed starting with 2009. All data were analyzed using the Statistical Package for Social Sciences software (version 16.0, SPSS, Inc.) and shown as mean \pm standard deviation. Graphs were plotted by Microsoft Excel 2008 (Microsoft Corporation). Means and standard deviations were calculated for all continuous variables. Frequency distribution and percentages were determined for categorical variables. Comparisons between post-operative and pre-operative values, for visual acuity (VA) and foveal thickness (FT) were carried out using the paired sample t-test. Concordance tests (Kolmogorov-Smirnov) have been performed for each data vector in order to determine their normality. For a 0.05 confidence interval, the data have been tested as normally 


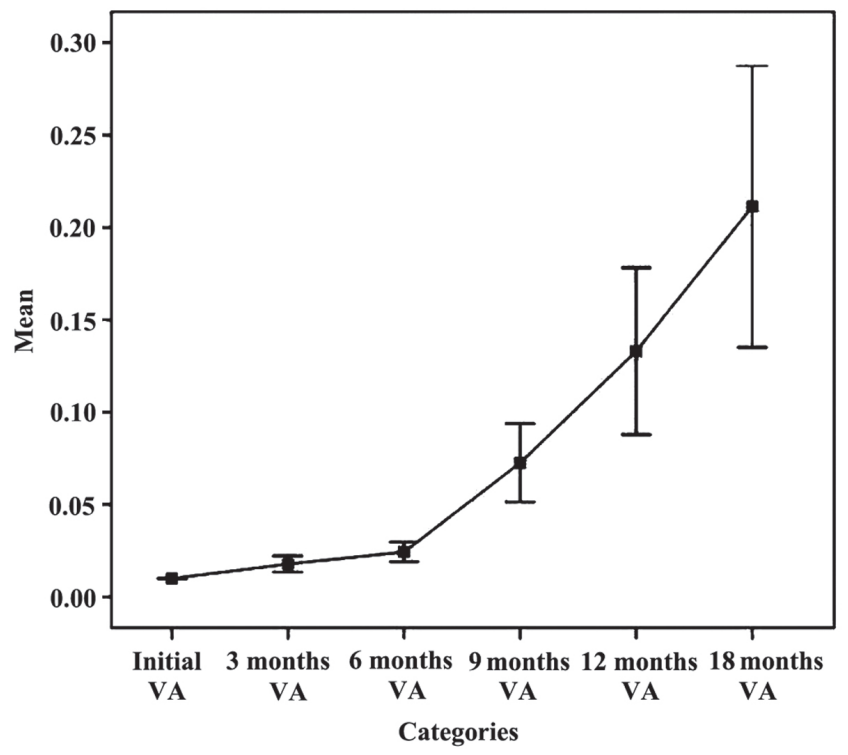

Figure 1. The evolution trend of the average VA.

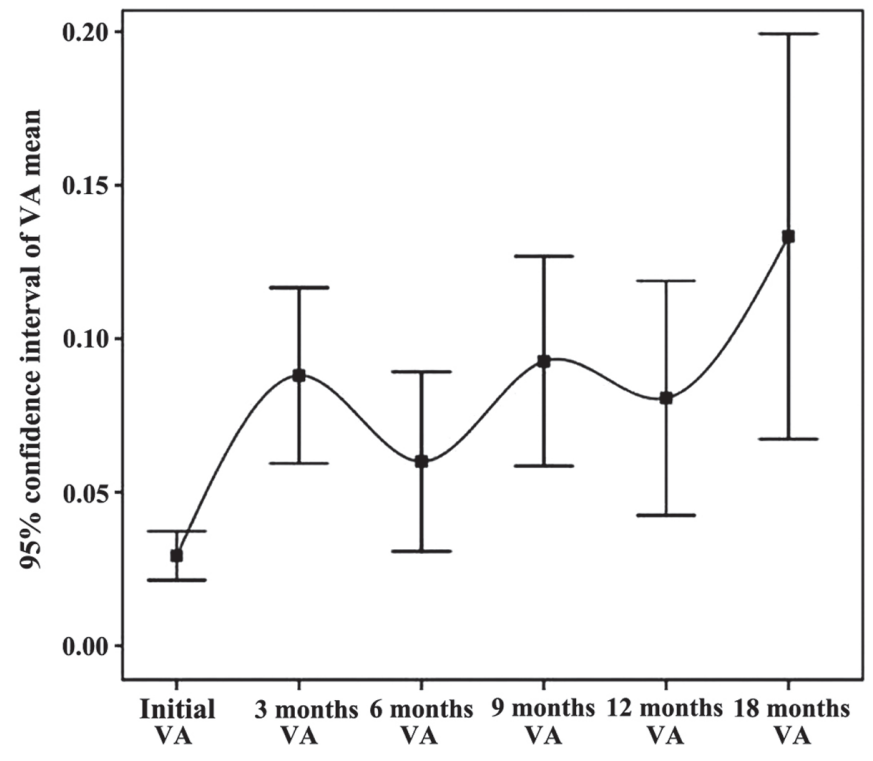

Figure 2. The evolution trend of the average VA in time.

distinguished from a bivariate approach where the visual acuity (VA) is correlated with foveal thickness (FT).

The analysis of VA parameter pursued independent batches of raw patients. The chart in Fig. 1 established an upward trend in mean parameter AV on intervals during follow-up. It looks favorable and functional, but statistically insignificant, due to alteration in time of lots followed and the remaining, not representative study subjects. Increasing the average parameter ranges VA 6 months - 9 months - 12 months - 18 months is an unreal situation. Practically, we planned and realized an improvement in visual acuity within 3 months and even within 6 months, maintaining relatively constant limits for longer periods of time (9, 12 or even 18 months), but failed, at least for now, to achieve both significant growth and progress over time. It is a gross analysis with rough results. For this reason, we cannot make quantitative judgments of value on the results of intravitreal bevacizumab therapy relying on such data, but we can make qualitative ones.

The analysis of VA parameter pursued independently on the lots processed and statistically adjusted by patients (Fig. 2). Preoperative visual acuity in the initial batch ranged from 0.01 to 0.5 . In the initial batch, mean visual acuity was 0.04 and after 3 months it was 0.12 . Visual acuity improved in the assessment of 3 months in 77 eyes (66\%), subjectively quantified by the patient or objectively by physician. In 40 eyes (34\%) there was no change in visual acuity. There was no case of decreased visual acuity postoperatively. In order to validate the statistical data, processing, adaptation and conformity tests were applied to patients' groups. The overall conclusion regarding visual acuity independent parameter (VA) is that it highlights a clear increasing trend of the parameter VA from the initial interval to 3 months, with a flat profile (maintaining a constant level) in the 3-, 6-, 9- and 12-month intervals.

The analysis of VA parameter pursued in conjunction with foveal thickness (FT). Analysis of the VA time intervals statistical analysis according to the two objectives proposed. Univariate results follow-up (referring only to VA) will be 
Table III. Average VA time intervals on the effect of functional environment and average thickness of the fovea per time intervals with medium structural effect.

\begin{tabular}{|c|c|c|c|c|}
\hline Time & Average VA & $\begin{array}{l}\text { Change in VA during treatment } \\
\text { from the previous stage }\end{array}$ & Average FT & $\begin{array}{l}\text { FT variation during treatment } \\
\text { from the initial stage }\end{array}$ \\
\hline Initial & 0.071 & & 450.941 & \\
\hline 1 month & 0.021 & 0.7 & & \\
\hline 2 months & 0.023 & -0.11 & & \\
\hline 3 months & 0.204 & 0.13 & 309.137 & -0.31 \\
\hline 6 months & 0.074 & -0.72 & 431.556 & 0.4 \\
\hline 9 months & 0.208 & -0.64 & 331.222 & -0.23 \\
\hline 12 months & 0.065 & 0.69 & 400.500 & 0.21 \\
\hline
\end{tabular}

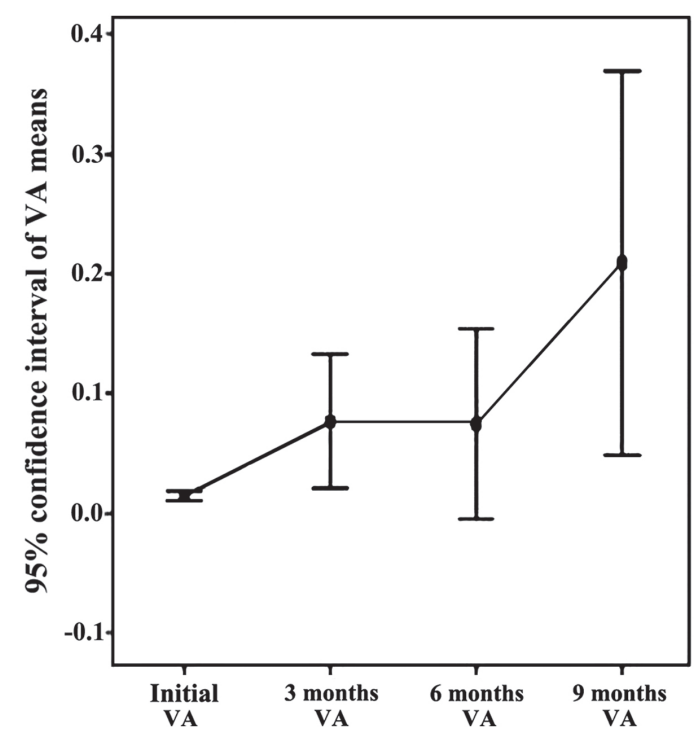

Figure 3. Increasing VA trend within the 9-month time interval.

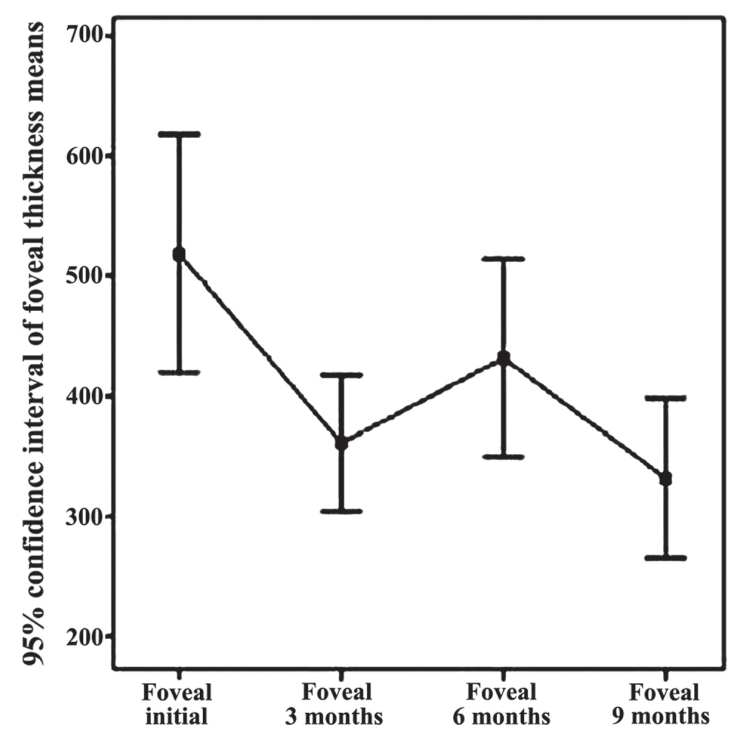

Figure 4. Decreasing FT trend within the 9-month time interval. highlighting the environmental functional effect and analysis of the average thickness of the fovea on time intervals to highlight the effect of structural environment (Table III, Figs. 3 and 4).

There is an ascending trend of visual acuity in baseline period, 9 months, due to the effect of bevacizumab injections (Fig. 3). Note the two slopes upward initially 3 months (by applying the standard protocol) and 6-9 months (by re-injection performed at 6 months). The appearance slightly decreasing on the 3-6 month interval is due to the reactivation of neovascular membranes.

In Fig. 4, a decrease of central retinal thickness in the baseline range of 9 months under the effect of intravitreal bevacizumab injection can be noted.

Fovea thickness (central retina), the initial batch, determined by OCT, ranged from 266 microns to 720 microns, with an average of $450.94( \pm 104.5)$ microns. Fovea thickness was significantly reduced following treatment with intravitreal bevacizumab, 3 months recorded an average of $309.13( \pm 66.57)$ microns. The overall conclusion regarding fovea thickness independent parameter refers to the downward trend in the range of parameter FT from initial to 3 months. In addition, it refers to an increase in central retinal thickness
(6 months), explained by recurrent exudative phenomena, again significantly reduced FT ( 9 months) through augmentation of antiangiogenic effect by re-injection of 6 months, as well as a relatively stable appearance of reference parameter within 9-12 months, item also followed and expected from the treatment.

The statistical analysis of the two parameters followed (visual acuity and foveal thickness) was statistically validated in an initial period of 9 months, the data gathered at 12 months and 18 months being inconsistent. The conclusion of this correlation is that for the initial time of 9 months, VA has an upward trend, while presenting a downward trend for FT in the pursued group, identifying a solid negative correlation between two variables for all periods included (initially 3 months, 3-6 months and 6-9 months).

The statistical analysis of the two followed parameters (visual acuity and foveal thickness) also showed that there were patients with choroidal neovascular membrane recurrence (VA reduction and FT increase) at varying intervals from baseline $(6,9$ or 12 months), for which re-injection option has led to significant improvement or stabilization of both structural and functional parameters. 


\section{Discussion}

Considerations on intravitreal bevacizumab use complications. Within the studied patient groups, we did not have systemic complications that could be associated with bevacizumab, such as cardiac or cerebral ischemia. There were, however, minor and local complications related to the technique of injection: subconjunctival hemorrhage, 31 cases of the 376 injections (8.2\%), corneal erosion, 2 cases $(0.5 \%)$, post-injection ocular surface discomfort such as: burning, stinging, mild pain at the injection site, itching, 11 cases $(2.9 \%)$. There were no serious local complications, such as retinal tears, rhegmatogenous retinal detachment, endophthalmitis, increased intraocular pressure, cataract, retinal pigment epithelium rupture or systemic complications like cardiac or cerebral thromboembolic accidents.

We have reviewed the results of prestigious research groups regarding pharmacokinetics, the effects on the blood-ocular barrier, electrophysiological effects, CNV histology, the general conclusion being that bevacizumab is not toxic at the currently used dose and can be used safely in patients with neovascular AMD (6-11).

Arguments and counter-arguments related to the personal study. In the series of cases diagnosed with choroidal neovascularization secondary to AMD and treated according to a personal algorithm with bevacizumab intravitreal injection, in the non-comparative retrospective analysis, there was a functional improvement from a quantitative and qualitative point of view (improvement of visual acuity, color perception optimizing, widening visual field, better appreciation of the spatial elements); in addition, good anatomical results (central retinal thickness reduction, reduction or disappearance of 'leakage') were also noted. The retrospective analysis of personal cases treated and followed was favorable to the use of intravitreal bevacizumab in short-term and medium treatment for choroidal neovascular membranes associated with AMD.

The study had several weaknesses: first of all, the design of the study - retrospective, non-randomized, non-controlled, interventional type on a number of cases - only allowed assessment of effectiveness treatment under subjective conditions chosen by the authors on a group of patients in whom they identified a common element, namely choroidal neovascularization associated with AMD. The absence of randomization and control group does not allow making proper interpretation of the epidemiological value. The 'selection' of cases based on clinical criteria (significant exudation, minimal fibrosis) allowed to obtain good results, situated somewhat above the average of other studies that used randomization and control elements. Another element inducing limits to the study is the presence of short-term follow-up data (3 months), reducing the database to track the medium term (9-12 months), but this represents a subjective aspect and it is difficult to control by the physician. In order to make valid judgments on the medium and long-term, the study group should not impair over time. The tests applied are of pair type (same subject is tested based on the same clinical parameters intervals and different conditions) always watching the number of subjects who remained in the study and therefore would not affect the representativeness and statistical significance of the results. For this reason, statistical analysis stopped at the 12 months interval, virtually ignoring the values recorded at 18 months.

Considerations regarding the use of intravitreal bevacizumab in treating neovascular AMD. Preclinical studies have suggested that full-length antibodies could not penetrate the retina and are therefore ineffective in the treatment of subretinal pathology, in this case choroidal neovascularization associated with AMD (12). The publication of clinical cases that showed an impressive resolution of the macula intraretinal fluid in Exudative AMD or Central Venous Occlusions have raised doubts over the previous term $(13,14)$. Subsequently, several studies have shown that bevacizumab molecule can penetrate the retina in the intravitreal administration $(6,15)$. A series of cases were also published showing favorable anatomic and functional effect of bevacizumab therapy in various ocular conditions associated with neovascular disease (16-20). The first clinical results showed a promising efficiency profile for Avastin in neovascular AMD $(21,22)$. There seems to be significant difference in terms of responsiveness to anti-VEGF between different types of choroidal neovascular membranes in classic or occult AMD, myopia, or juxtafoveal telangiectasis and angioid streaks (21,23-31).

The intravitreal injection rate of adverse effects for bevacizumab did not exceed that observed in control groups from trials with ophthalmology accepted anti-VEGF (ranibizumab). There was no more than $0.21 \%$ incidence of any adverse effect on an average follow-up period of 3.5 months.

In cconclusion, the increased prevalence of wet AMD, along with the severity of functional visual impairment have led to significant research concentration on all issues identified in correlation with the background condition. In the last thirteen years of ophthalmology, there was a huge explosion belonging both to the anti-angiogenic agents in the usual therapeutic arsenal and to the optical coherence tomography as a standard tool of diagnosis. The introduction of intravitreal bevacizumab in Romania has also led to a fantastic increase in number of OCT equipment, from four units in 2006 to more than two hundred. The huge potential of anti-VEGF medication is revealed more and more while allowing the development of new therapeutic principles and new classes of drugs. Although treatment with anti-VEGF first addressed choroidal neovascular membranes from AMD, the range of therapeutic indications of this new class of substances was expanded considerably to other pathologies (16-18,32-38).

To the best of our knowledge, this is the first study in Romania that assessed, analyzed and correlated clinical and statistically functional (improvement of visual acuity) and structural (central retinal thickness reduction) results of intravitreal bevacizumab treatment in patients with neovascular AMD, while comparing them with results of baseline studies in literature on the same topic.

We have been using bevacizumab in our daily practice for thirteen years and we have decided to publish our results from the beginning of our own experience (2006) as a tribute to the remarkable results of this efficient, safe and accessible drug for wet AMD patients. By using anti-VEGF agents in 
our clinical approach, we could improve and prolong visual function for many patients who did not have any viable option till antiangiogenics was born. Bevacizumab is an affordable treatment when other, more expensive anti-VEGF agents, are not available. Soon, there will probably be new options at our disposal, aiming to overcome macular atrophy and also subretinal fibrosis, items which bevacizumab could not address. As physicians, our goal is to offer the best medical solution to our patients; thus, we must continuously prepare to fight against 'unresolved' issues.

\section{Acknowledgements}

Professional editing, linguistic and technical assistance performed by Individual Service Provider Irina Radu, certified translator in Medicine and Pharmacy.

\section{Funding}

Not applicable.

\section{Availability of data and materials}

All data and materials supporting the results of the present study are available in the article.

\section{Authors' contributions}

HTS contributed to the conception and design of the study, the acquisition, analysis and interpretation of data of the study. Also contributed in drafting the work and revising it critically for important intellectual content. SS contributed to the design of the study and in drafting the study and revising it critically for important intellectual content. BT and MD contributed to the acquisition, analysis and interpretation of data of the study and in the drafting the work. FB contributed to the conception and design of the study and in revising the work critically for important intellectual content. All authors read and approved the final version of the manuscript and agreed to be accountable for all aspects of the study in ensuring that questions related to the accuracy or integrity of any part of the work are appropriately investigated and resolved.

\section{Ethics approval and consent to participate}

This study adhered to the tenets of the Declaration of Helsinki and was approved by the Ethics Committee of our hospital, 'Prof. Dr. Agrippa Ionescu' Clinical Emergency Hospital. Written informed consent was obtained from all the patients before the examinations and surgeries were performed.

\section{Patient consent for publication}

Not applicable.

\section{Competing interests}

The authors declare that they have no competing interests.

\section{References}

1. Klein R, Klein BE, Jensen SC and Meuer SM: The five-year incidence and progression of age-related maculopathy: The Beaver Dam Eye Study. Ophthalmology 104: 7-21, 1997.

2. National Eye Institute: Statistics and Data. Prevalence of Blindness Data. Data Tables [online] 2007. https://nei.nih.gov/ eyedata/pbd_tables.

3. Fine SL, Berger JW, Maguire MG and Ho AC: Age-related macular degeneration. N Engl J Med 342: 483-492, 2000.

4. Ciulla TA,Harris A and Martin BJ: Ocularperfusion and age-related macular degeneration. Acta Ophthalmol Scand 79: 108-115, 2001.

5. Moshfeghi AA, Rosenfeld PJ, Puliafito CA, Michels S, Marcus EN, Lenchus JD and Venkatraman AS: Systemic bevacizumab (Avastin) therapy for neovascular age-related macular degeneration: Twenty-four-week results of an uncontrolled open-label clinical study. Ophthalmology 113: 2002.e1-2002.e12, 2006.

6. Rosenfeld PJ, Fung AE and Puliafito CA: Optical coherence tomography findings after an intravitreal injection of bevacizumab (avastin) for macular edema from central retinal vein occlusion. Ophthalmic Surg Lasers Imaging 36: 336-339, 2005.

7. Bakri SJ, Cameron JD, McCannel CA, Pulido JS and Marler RJ Absence of histologic retinal toxicity of intravitreal bevacizumab in a rabbit model. Am J Ophthalmol 142: 162-164, 2006.

8. Manzano RP, Peyman GA, Khan P and Kivilcim M: Testing intravitreal toxicity of bevacizumab (Avastin). Retina 26: 257-261, 2006

9. Kiss C, Michels S, Prager F, Weigert G, Geitzenauer W and Schmidt-Erfurth U: Anterior chamber inflammatory activity in patients with neovascular age-related macular degeneration treated with intravitreal bevacizumab (Avastin). Retina 26: 877-881, 2006

10. Spitzer MS, Wallenfels-Thilo B, Sierra A, Yoeruek E, Peters S, Henke-Fahle S, Bartz-Schmidt KU and Szurman P; Tuebingen Bevacizumab Study Group: Antiproliferative and cytotoxic properties of bevacizumab on different ocular cells. Br J Ophthalmol 90: 1316-1321, 2006.

11. Ziemssen F, Warga M, Neuhann IM, Leitritz M, Biester S, Grisanti S and Bartz-Schmidt KU: Does intravitreal injection of bevacizumab have an effect on the blood-aqueus barrier function? Br J Ophthalmol 90: 922, 2006.

12. Mangione CM, Lee PP, Gutierrez PR, Spritzer K, Berry S, Hays RD; National Eye Institute Visual Function Questionnaire Field Test Investigators: Development of the 25-item National Eye Institute Visual Function Questionnaire. Arch Ophthalmol 119: 1050-1058, 2001

13. Mordenti J, Cuthbertson RA, Ferrara N, Thomsen K, Berleau L, Licko V, Allen PC, Valverde CR, meng YG, Fei DT, et al: Comparisons of the intraocular tissue distribution, pharmacokinetics, and safety of ${ }^{125}$ I-labeled full-length and Fab antibodies in rhesus monkeys following intravitreal administration. Toxicol Pathol 27: 536-544, 1999.

14. Rosenfeld PJ, Moshfeghi AA and Puliafito CA: Optical coherence tomography findings after an intravitreal injection of bevacizumab (avastin) for neovascular age-related macular degeneration. Ophthalmic Surg Lasers Imaging 36: 331-335, 2005.

15. Shahar J, Avery RL, Heilweil G, Barak A, Zemel E, Lewis GP, Johnson PT, Fisher SK, Perlman I and Loewenstein A: Electrophysiologic and retinal penetration studies following intravitreal injection of bevacizumab (Avastin). Retina 26: 262-269, 2006

16. Spaide RF, Laud K, Fine HF, Klancnik JM Jr, Meyerle CB, Yannuzzi LA, Sorenson J, Slakter J, Fisher YL and Cooney MJ: Intravitreal bevacizumab treatment of choroidal neovascularization secondary to age-related macular degeneration. Retina 26: 383-390, 2006

17. Heiduschka P, Fietz H, Hofmeister S, Schultheiss S, Mack AF, Peters S, Ziemssen F, Niggemann B, Julien S, Bartz-Schmidt KU, et al; Tübingen Bevacizumab Study Group: Penetration of bevacizumab through the retina after intravitreal injection in the monkey. Invest Ophthalmol Vis Sci 48: 2814-2823, 2007

18. Iturralde D, Spaide RF, Meyerle CB, Klancnik JM, Yannuzzi LA, Fisher YL, Sorenson J, Slakter JS, Freund KB, Cooney M, et al: Intravitreal bevacizumab (Avastin) treatment of macular edema in central retinal vein occlusion: A short-term study. Retina 26: 279-284, 2006

19. Spaide RF and Fisher YL: Intravitreal bevacizumab (Avastin) treatment of proliferative diabetic retinopathy complicated by vitreous hemorrhage. Retina 26: 275-278, 2006. 
20. Avery RL: Regression of retinal and iris neovascularization after intravitreal bevacizumab (Avastin) treatment. Retina 26: 352-354, 2006.

21. Avery RL, Pearlman J, Pieramici DJ, Rabena MD, Castellarin AA, Nasir MA, Giust MJ, Wendel R and Patel A: Intravitreal bevacizumab (Avastin) in the treatment of proliferative diabetic retinopathy. Ophthalmology 113: 1695.e1-1695.e15, 2006.

22. Aisenbrey S, Ziemssen F, Völker M, Gelisken F, Szurman P, Jaissle G, Grisanti S and Bartz-Schmidt KU: Intravitreal bevacizumab (Avastin) for occult choroidal neovascularization in age-related macular degeneration. Graefes Arch Clin Exp Ophthalmol 245: 941-948, 2007.

23. Avery RL, Pieramici DJ, Rabena MD, Castellarin AA, Nasir MA and Giust MJ: Intravitreal bevacizumab (Avastin) for neovascular age-related macular degeneration. Ophthalmology 113: 363-372. e5, 2006.

24. Yoganathan P, Deramo VA, Lai JC, Tibrewala RK and Fastenberg DM: Visual improvement following intravitreal bevacizumab (Avastin) in exudative age-related macular degeneration. Retina 26: 994-998, 2006.

25. Sakaguchi H, Ikuno Y, Gomi F, Kamei M, Sawa M, Tsujikawa M, Oshima Y, Kusaka S and Tano Y: Intravitreal injection of bevacizumab for choroidal neovascularisation associated with pathological myopia. Br J Ophthalmol 91: 161-165, 2007.

26. Laud K, Spaide RF, Freund KB, Slakter J and Klancnik JM Jr: Treatment of choroidal neovascularization in pathologic myopia with intravitreal bevacizumab. Retina 26: 960-963, 2006.

27. Tewari A, Dhalla MS and Apte RS: Intravitreal bevacizumab for treatment of choroidal neovascularization in pathologic myopia. Retina 26: 1093-1094, 2006.

28. Teixeira A, Moraes N, Farah ME and Bonomo PP: Choroidal neovascularization treated with intravitreal injection of bevacizumab (Avastin) in angioid streaks. Acta Ophthalmol Scand 84: 835-836, 2006

29. Lommatzsch A, Spital G, Trieschmann M and Pauleikhoff D: Intraocular application of bevacizumab for the treatment of choroidal neovascularization secondary to angioid streaks. Ophthalmologe 104: 325-328, 2007 (In German).

30. Jorge R, Costa RA, Calucci D and Scott IU: Intravitreal bevacizumab (Avastin) associated with the regression of subretinal neovascularization in idiopathic juxtafoveolar retinal telangiectasis. Graefes Arch Clin Exp Ophthalmol 245: 1045-1048, 2007.
31. Munteanu M and Rosca C: Repositioning and follow-up of intralenticular dexamethasone implant. J Cataract Refract Surg 39: 1271-1274, 2013

32. Jianu DC, Jianu SN, Dan TF, Motoc AGM and Poenaru M Pulsatile tinnitus caused by a dilated left petrosquamosal sinus. Rom J Morphol Embryol 57: 319-322, 2016.

33. Stanca HT, Suvac E, Munteanu M, Jianu DC, Motoc AGM, Rosca GC and Boruga O: Giant cell arteritis with arteritic anterior ischemic optic neuropathy. Rom J Morphol Embryol 58: 281-285, 2017.

34. Caruntu C, Boda D, Dumitrascu G, Constantin C and Neagu M: Proteomics focusing on immune markers in psoriatic arthritis. Biomarkers Med 9: 513-528, 2015.

35. Solomon I, Voiculescu VM, Caruntu C, Lupu M, Popa A, Ilie MA, Albulescu R, Caruntu A, Tanase C, Constantin C, et al: Neuroendocrine factors and head and neck squamous cell carcinoma: An affair to remember. Dis Markers 2018: 9787831, 2018.

36. Ion A, Popa IM, Papagheorghe LML, Lisievici C, Lupu M, Voiculescu V, Caruntu C and Boda D: Proteomic approaches to biomarker discovery in cutaneous T-cell lymphoma. Dis Markers 2016: 9602472, 2016.

37. Lupu M, Caruntu A, Caruntu C, Papagheorghe LML, Ilie MA Voiculescu V, Boda D, Constantin C, Tanase C, Sifaki M, et al: Neuroendocrine factors: The missing link in non-melanoma skin cancer (Review). Oncol Rep 38: 1327-1340, 2017.

38. Batani A, Brănișteanu DE, Ilie MA, Boda D, Ianosi S, Ianosi G and Caruntu C: Assessment of dermal papillary and microvascular parameters in psoriasis vulgaris using in vivo reflectance confocal microscopy. Exp Ther Med 15: 1241-1246, 2018.

This work is licensed under a Creative Commons Attribution-NonCommercial-NoDerivatives 4.0 International (CC BY-NC-ND 4.0) License. 